\title{
Connectivity properties of moment maps on based loop groups
}

\author{
Megumi HaRAda \\ TARA S HOLM \\ LISA C JEFFREY \\ Augustin-Liviu Mare
}

\begin{abstract}
For a compact, connected, simply-connected Lie group $G$, the loop group $L G$ is the infinite-dimensional Hilbert Lie group consisting of $H^{1}$-Sobolev maps $S^{1} \rightarrow G$. The geometry of $L G$ and its homogeneous spaces is related to representation theory and has been extensively studied. The space of based loops $\Omega(G)$ is an example of a homogeneous space of $L G$ and has a natural Hamiltonian $T \times S^{1}$ action, where $T$ is the maximal torus of $G$. We study the moment map $\mu$ for this action, and in particular prove that its regular level sets are connected. This result is as an infinite-dimensional analogue of a theorem of Atiyah that states that the preimage of a moment map for a Hamiltonian torus action on a compact symplectic manifold is connected. In the finite-dimensional case, this connectivity result is used to prove that the image of the moment map for a compact Hamiltonian $T$-space is convex. Thus our theorem can also be viewed as a companion result to a theorem of Atiyah and Pressley, which states that the image $\mu(\Omega(G))$ is convex. We also show that for the energy functional $E$, which is the moment map for the $S^{1}$ rotation action, each non-empty preimage is connected.
\end{abstract}

53D20; 22E65

\section{Introduction}

The main results of this paper are infinite-dimensional analogues of well-known results in finite-dimensional symplectic geometry. More specifically, given a compact connected Hamiltonian $T$-manifold with moment map $\mu: M \rightarrow \mathrm{t}^{*}$, a theorem of Atiyah [1, Theorem 1] states that any non-empty preimage of $\mu$ is connected. This connectivity result is intimately related to the famous convexity result of Atiyah and Guillemin-Sternberg $[1 ; 7]$, that states that the image $\mu(M) \subseteq \mathfrak{t}^{*}$ is convex. Indeed, in the original paper of Atiyah, the connectivity result is used to establish the convexity of $\mu(M)$. 
Atiyah and Pressley [2] showed that the convexity results mentioned above generalize to a certain class of infinite-dimensional manifolds that are homogeneous spaces of loop groups $L G$. An example is illustrated in Figure 1. Indeed, there is a general principle (see eg, Pressley and Segal $[25 ; 26]$ ) which says that these infinite-dimensional homogeneous spaces of $L G$ behave, in many respects, like compact Kähler manifolds. The result of [2] may be viewed as an instance of this, as should the results of this paper. However, in contrast to the argument given in [1], the convexity result in [2] is proven without any mention of the connectivity of level sets of the moment map. Therefore a natural question still remains: is there an analogous connectivity result for these homogeneous spaces of loop groups? The purpose of this paper is to answer this question in the affirmative.

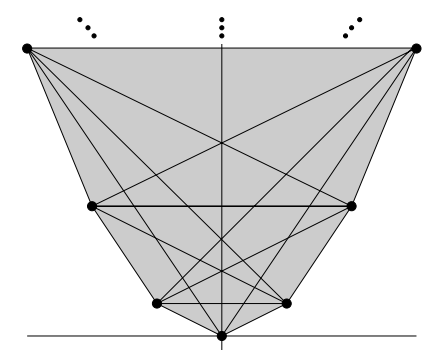

Figure 1: The shaded region indicates a portion of the image in $\left(\mathfrak{t}^{2}\right)^{*}$ of the moment map for the $T^{2}=T^{1} \times S^{1}$ action on $\Omega(S U(2))$, which is the convex hull of the integer points on a parabola. (We have also drawn in the critical values of $\mu$, which are the line segments.) The convexity of the image was proven in [26]. We will show that the level sets of this map are connected.

In the finite-dimensional setting, connectivity of the level sets has several important implications. Some of these suggest very natural questions for the infinite-dimensional context; we mention three of these here. First, connectivity along with a local normal form description of the level sets yields the Atiyah-Guillemin-Sternberg convexity result. A new proof of the Atiyah-Pressley convexity result for $\Omega G$ that uses our connectivity result and some additional information about the local normal forms would be very interesting. Second, connectivity is the first piece of Kirwan's surjectivity theorem [13]. Given a compact connected Hamiltonian $G$-manifold with moment map $\mu: M \rightarrow \mathfrak{g}^{*}$, Kirwan showed that the inclusion of a level set $\mu^{-1}(0) \hookrightarrow M$ induces a surjection in equivariant cohomology. If the level set is regular, its equivariant cohomology is isomorphic to the ordinary cohomology of the symplectic quotient. Thus, there is a surjection $H_{G}^{*}(M) \rightarrow H^{*}(M / / G)$. For the homogeneous spaces of loop groups, connectivity of the level sets implies that this map is a surjection for 
degree 0 cohomology. A surjection $\kappa: H_{T \times S^{1}}^{*}(\Omega G) \rightarrow H^{*}\left(\Omega G / / T \times S^{1}\right)$ would be a very powerful result. By understanding the map $\kappa$ and its kernel, and using the description of $H_{T \times S^{1}}^{*}(\Omega G)$ in Harada, Henriques and Holm [9], one could obtain a description of the cohomology ring of this quotient. Extending known results about $\kappa$ and its kernel (eg, Jeffrey, Kirwan, Tolman and Weitsman [11;29]) will nevertheless require significant technical prowess. Moreover, there is a possible interpretation in terms of representation theory. According to the so-called "quantization commutes with reduction" principle in the finite-dimensional setting, it may be possible to interpret the spaces $\Omega G / / T \times S^{1}$ as geometric analogues of $T \times S^{1}$-weight spaces for certain loop group representations. Third, in finite dimensions, Karshon and Lerman [12] used the connectivity of level sets to deal with the double commutator conjecture of Guillemin and Sternberg [8]. This conjecture states that the centralizer of the algebra of $G$-invariant functions on a Hamiltonian $G$-manifold is collective, ie, the pullback via the $G$-moment map of a smooth function on $\mathfrak{g}^{*}$. The connectivity results contained herein may aid in the determining the validity of an infinite dimensional analogue of this theorem.

We now state more precisely the main results of this paper. Let $G$ be a compact, connected and simply-connected Lie group. We fix a $G$-invariant inner product $\langle$, on the Lie algebra $\mathfrak{g}$. Let $L_{1}(G)$ denote the space of all maps $S^{1} \cong \mathbb{R} / 2 \pi \mathbb{Z} \rightarrow G$ that are of Sobolev class $H^{1}$, ie,

$$
L_{1}(G):=\left\{\eta \in H^{1}\left(S^{1}, G\right)\right\} .
$$

Remark Throughout the remainder of this paper, $H^{1}$ stands always for "Sobolev class" (and not for "cohomology").

The space $L_{1}(G)$ is a group by pointwise multiplication. We now consider the subgroup $\Omega_{1}(G)$ of $L_{1}(G)$ consisting of based loops $\gamma$, ie, we add the requirement $\gamma(0)=e \in G$. The group $L_{1}(G)$ acts by conjugation on $\Omega_{1}(G)$ and it is straightforward to see that the stabilizer of the constant loop at $e$ is exactly the set $G \subseteq L_{1}(G)$ of constant loops. Hence $\Omega_{1}(G)$ is a homogeneous space $L_{1}(G) / G$. This is a smooth Hilbert manifold (see eg, Palais [23]) which is also Kähler (see Atiyah, Pressley and Segal [2; 26]), and is the space on which we will focus for the remainder of the paper.

We consider the following torus action on $\Omega_{1}(G)$. The maximal torus $T$ of $G$ is a subgroup of $L_{1}(G)$, and hence acts on the left on $\Omega_{1}(G) \cong L_{1}(G) / G$. More specifically, for $t \in T, \gamma \in \Omega_{1}(G)$, the action is defined by

$$
(t \gamma)(\theta):=t \gamma(\theta) t^{-1} \text {. }
$$


In addition to this maximal torus action, we also have a rotation action of $S^{1}$ that rotates the loop variable. For $e^{i s} \in S^{1}$ and $\gamma \in \Omega_{1}(G)$, we have

$$
\left(e^{i s} \gamma\right)(\theta):=\gamma(s+\theta) \gamma(s)^{-1} \text {. }
$$

These actions commute, so we obtain a $T \times S^{1}$ action on $\Omega_{1}(G)$ which is also Hamiltonian with respect to the Kähler structure on $\Omega_{1}(G)$. The $S^{1}$-moment map is in fact a well-known functional on spaces of loops: it is the energy functional

$$
E(\gamma):=\frac{1}{4 \pi} \int_{0}^{2 \pi}\left\|\gamma(\theta)^{-1} \gamma^{\prime}(\theta)\right\|^{2} d \theta
$$

The moment map for the $T$-action is given by a similar functional,

$$
p(\gamma)=\operatorname{pr}_{\mathfrak{t}}\left(\frac{1}{2 \pi} \int_{0}^{2 \pi} \gamma(\theta)^{-1} \gamma^{\prime}(\theta) d \theta\right),
$$

where $\mathfrak{t}:=\operatorname{Lie}(T)$ and $\mathrm{pr}_{\mathfrak{t}}: \mathfrak{g} \rightarrow \mathfrak{t}$ denotes the orthogonal projection with respect to the fixed $G$-invariant inner product $\langle\cdot, \cdot\rangle$. Using this inner product, we may identify $\mathfrak{t}^{*}$ with $\mathfrak{t}$, and use the standard inner product on $\mathbb{R}$ to identify $\mathbb{R}^{*}$ with $\mathbb{R}$. With these identifications, the $T \times S^{1}$-moment map $\mu: \Omega_{1}(G) \rightarrow \mathfrak{t}^{*} \oplus \mathbb{R}^{*} \cong \mathfrak{t} \oplus \mathbb{R}$ is given by $\mu=p \oplus E$.

There is a subspace of $\Omega_{1}(G)$ that we will also study, namely $\Omega_{\mathrm{alg}}(G)$, which is the space of algebraic loops in $G$. The main result of [2], as mentioned above, is that the images $\mu\left(\Omega_{\mathrm{alg}}(G)\right)$ and $\mu\left(\Omega_{1}(G)\right)$ are both convex. Our results are as follows.

Theorem 1.1 Any level of $\mu: \Omega_{\mathrm{alg}}(G) \rightarrow \mathfrak{t} \oplus \mathbb{R}$ is connected or empty.

Theorem 1.2 Any regular level of $\mu: \Omega_{1}(G) \rightarrow \mathfrak{t} \oplus \mathbb{R}$ is connected or empty.

We also have a statement of connectivity for the level sets of just the energy functional, considered as a function on $\Omega_{1}(G)$ or on $\Omega_{\mathrm{alg}}(G)$. In this case, we have the connectivity for any (possibly singular) value of $E$.

Theorem 1.3 For $\bullet \in\{1$, alg $\}$, all preimages of $E: \Omega_{\bullet}(G) \rightarrow \mathbb{R}$ are either empty or connected.

We now make explicit the topologies on the spaces $\Omega_{\bullet}(G)$ with respect to which we state our Theorems 1.1-1.3 above. First, $\Omega_{1}(G)$ has a natural topology induced from $H^{1}\left(S^{1}, \mathfrak{g}\right)$ via the exponential map exp: $\mathfrak{g} \rightarrow G$, which is a local homeomorphism around 0 (see [2, Definition 2.4]). The issue of the topology on the subspace $\Omega_{\text {alg }}(G)$ is more subtle. On the one hand, $\Omega_{\mathrm{alg}}(G)$ is equipped with the subset topology induced 
from the inclusion $\Omega_{\mathrm{alg}}(G) \hookrightarrow \Omega_{1}(G)$. However, $\Omega_{\mathrm{alg}}(G)$ also has a stratification by algebraic varieties, which gives it a direct limit topology (see [26, Section 3.5]). For Theorems 1.1 and 1.3 above, we consider $\Omega_{\mathrm{alg}}(G)$ with the direct limit topology. The two topologies on $\Omega_{\mathrm{alg}}(G)$ are related; the subset topology is coarser than the direct limit topology (see eg, Proposition 2.1), so Theorem 1.1 in fact implies that $\mu^{-1}(a) \cap \Omega_{\mathrm{alg}}(G)$ is connected with respect to either of the possible topologies of $\Omega_{\text {alg }}(G)$.

It is worth remarking here that the proofs of connectivity for $\Omega_{\mathrm{alg}}(G)$ and $\Omega_{1}(G)$ are markedly different in flavor. To prove connectivity of levels for both $\mu$ and $E$ on $\Omega_{\mathrm{alg}}(G)$, we exploit the algebraic structure of $\Omega_{\mathrm{alg}}(G)$, in particular that there it may be described as a union of finite-dimensional subvarieties. The proofs of the connectivity results for $\Omega_{1}(G)$, on the other hand, use the connectivity results for $\Omega_{\text {alg }}(G)$ and the density (in the $H^{1}$ topology) of $\Omega_{\text {alg }}(G)$ as a subset of $\Omega_{1}(G)$. The tool used here is Morse theory in infinite dimensions. To use Morse theory in this context requires additional technical hypotheses, such as the Palais-Smale condition (C). These hypotheses must explicitly be checked in order for us to use the Morse-theoretic arguments, and these are the main technical difficulties in this paper.

We now outline the contents of this manuscript. In Section 2 we briefly recall important known results about $\Omega_{\bullet}(G)$ and its moment maps. Then in Section 3 we prove the result for algebraic loops $\Omega_{\text {alg }}(G)$. Section 4 is devoted to the proof of Theorem 1.2. The argument in Sections 3 and 4 also proves certain cases of Theorem 1.3. Finally, in Section 5, we prove Theorem 1.3 for the case of the singular levels of the energy functional on $\Omega_{1}(G)$, which requires a separate argument.

Remark It would be interesting to extend Theorem 1.1 and Theorem 1.2 to arbitrary adjoint orbits of Kac-Moody groups or even to isoparametric submanifolds in Hilbert space (cf Terng and Mare [28; 20]).

Acknowledgments We would like to thank M Brion, R Cohen, Y Karshon, Y-H Kiem, E Lerman, R Sjamaar and J Woolf for helpful discussions. We also thank the American Institute of Mathematics for hosting all four authors while some of this work was conducted. The second author was supported in part by a National Science Foundation Postdoctoral Fellowship. The third and fourth authors are supported in part by NSERC.

\section{Background material}

In this section we collect facts that will be needed in the proofs of our main results. 


\subsection{Loop groups}

The main reference for this section is [2], especially section 2 (see also Freed [5, Section 1] or Pressley [25, Section 2]). By definition, $L_{1}(G)=H^{1}\left(S^{1}, G\right)$ is the space of free loops in $G$ of Sobolev class $H^{1}$. These are maps $\eta: S^{1} \rightarrow G$ with the property that for any local coordinate system $\Phi$ on $G$, the map $\Phi \circ \eta: S^{1} \rightarrow \mathbb{R}^{\operatorname{dim} G}$ is of Sobolev class $H^{1}$. The space $L_{1}(G)$ is an infinite dimensional Lie group (cf [23, Section 13]). It carries a natural left invariant Riemannian metric, which will be addressed as the $H^{1}$ metric. This is uniquely determined by its restriction to the Lie algebra of $L_{1}(G)$, which is $H^{1}\left(S^{1}, \mathfrak{g}\right)$ (the tangent space at the constant loop $e$ ). First we fix an $\operatorname{Ad}(G)$-invariant metric on the Lie algebra $\mathfrak{g}$, denoted by $($,$) . The H^{1}$ metric is determined by

$$
\langle\gamma, \eta\rangle_{e}=\frac{1}{2 \pi} \int_{0}^{2 \pi}(\gamma(\theta), \eta(\theta)) d \theta+\frac{1}{2 \pi} \int_{0}^{2 \pi}\left(\gamma^{\prime}(\theta), \eta^{\prime}(\theta)\right) d \theta,
$$

for $\gamma, \eta \in H^{1}\left(S^{1}, \mathfrak{g}\right)$.

As mentioned in the introduction, the object of study of our paper is the space $\Omega_{1}(G)$. This is a closed submanifold of $L_{1}(G)$ and the $H^{1}$ metric defined above induces a metric on $\Omega_{1}(G)$, which we will denote by the same symbol $\langle$,$\rangle (the standard$ reference for this is [23, Section 13 , especially Theorem (6)]). We will also consider the Kähler metric on $\Omega_{1}(G)$. The details of the construction of this metric can be found in the references indicated at the beginning of the section (see also [26, Section 8.9]). We will just mention here that $\Omega_{1}(G)$ carries a natural symplectic form $\omega$, which is $L_{1}(G)$-invariant and its value at $e$ (the constant loop at the identity) is

$$
\omega_{e}(\gamma, \eta)=\frac{1}{2 \pi} \int_{0}^{2 \pi}\left(\gamma^{\prime}(\theta), \eta(\theta)\right) d \theta .
$$

There is a certain complex structure $J$ on $\Omega(G)$ and it can be shown that the triple $\left(\Omega_{1}(G), \omega, J\right)$ is a Kähler (Hilbert) manifold (see [26, Proposition 8.9.8]). The corresponding Kähler metric will be denoted by $g$ in our paper. The difference between the $H^{1}$ and the Kähler metric is that the first one is complete whereas the second one is not (this observation will play an important role in Section 4). To understand where the difference comes from, we note that both metrics are induced by the closed embedding $\Omega_{1}(G) \subset L_{1}(G)$ (see above). The $H^{1}$ metric on $L_{1}(G)=H^{1}\left(S^{1}, G\right)$ is obviously complete. The Kähler metric on $\Omega_{1}(G)$ is the restriction of the $H^{\frac{1}{2}}$ metric on $L_{1}(G)$ (this was first noted by Pressley in [25, Section 2]), hence it is not complete. For a detailed discussion about Sobolev metrics on loop groups, we refer the reader to [5, Section 1]. 
Without loss of generality, we may assume that $G$ is a closed subgroup of $S U(N)$, for $N$ sufficiently large. Moreover, we may also assume that the torus $T$ consists of diagonal matrices in $S U(N)$. It can be seen [23, Section 13] that $\Omega_{1}(G)$ is the space of all maps $\gamma$ of class $H^{1}$ from $S^{1}=\mathbb{R} / 2 \pi \mathbb{Z}$ to the space $M^{N \times N}(\mathbb{C})$ of all complex $N \times N$ matrices that have the properties that $\gamma\left(S^{1}\right) \subset G$ and $\gamma(0)$ is the identity matrix $I$. Morover, $\Omega_{1}(G)$ is a submanifold of

$$
L_{1}\left(M^{N \times N}(\mathbb{C})\right):=H^{1}\left(S^{1}, M^{N \times N}(\mathbb{C})\right) .
$$

Any $\gamma \in \Omega_{1}(G)$ has a Fourier expansion with coefficients in $M^{N \times N}(\mathbb{C})$. Then the subspace $\Omega_{\mathrm{alg}}(G)$ is defined to be the set of all algebraic loops, ie, loops $\gamma$ with finite Fourier series

$$
\gamma(\theta)=\sum_{k=-n}^{n} A_{k} e^{i k \theta},
$$

where $A_{k} \in M^{N \times N}(\mathbb{C})$, and $n \in \mathbb{Z}$. Consider the space

$$
L_{\mathrm{alg}}\left(M^{N \times N}(\mathbb{C})\right):=\left\{\gamma: S^{1} \rightarrow M^{N \times N}(\mathbb{C}): \gamma \text { is a finite Fourier series }\right\} .
$$

For any integer $n \geq 0$, we denote by $L_{n}$ the set of all elements of $L_{\text {alg }}\left(M^{N \times N}(\mathbb{C})\right)$ of the form (2-1). The space $L_{n}$ can be identified naturally with the direct product $\left(M^{N \times N}(\mathbb{C})\right)^{2 n+1}$, hence it carries a natural topology. It is obvious that the spaces $L_{0}, L_{1}, \ldots$ are a filtration of $L_{\mathrm{alg}}\left(M^{N \times N}(\mathbb{C})\right)$, in the sense that

$$
L_{0} \subset L_{1} \subset \ldots \subset L_{n} \subset \ldots L_{\mathrm{alg}}\left(M^{N \times N}(\mathbb{C})\right),
$$

and

$$
\bigcup_{j \geq 0} L_{j}=L_{\mathrm{alg}}\left(M^{N \times N}(\mathbb{C})\right)
$$

In this way, $L_{\mathrm{alg}}\left(M^{N \times N}(\mathbb{C})\right)$ can be equipped with the direct limit topology (by definition, a subset $U \subset L_{\mathrm{alg}}\left(M^{N \times N}(\mathbb{C})\right)$ is open iff $U \cap L_{j}$ is open in $L_{j}$ for any $j \geq 0)$. The topology induced on $\Omega_{\mathrm{alg}}(G)=\Omega_{1}(G) \cap L_{\mathrm{alg}}\left(M^{N \times N}(\mathbb{C})\right)$ will be also called the direct limit topology. Now $\Omega_{\mathrm{alg}}(G)$ inherits a topology from $\Omega_{1}(G)$ as well. This will be addressed as the subspace topology. The following result seems to be known (see eg, [6]). We include a proof for the sake of completeness (we will use this result in Section 4).

Proposition 2.1 The direct limit topology on $\Omega_{\mathrm{alg}}(G)$ is finer than the subspace topology. 
Proof In order to prove the lemma, it is sufficient to prove that the direct limit topology is finer than (the restriction of the) $H^{1}$ topology on $L_{\text {alg }}\left(M^{N \times N}(\mathbb{C})\right)$. Thus we need to prove that if $\gamma_{n} \in L_{\mathrm{alg}}\left(M^{N \times N}(\mathbb{C})\right)$ and $\left(\gamma_{n}\right)$ converges to 0 in the direct limit topology, then $\left(\gamma_{n}\right)$ converges to 0 in the $H^{1}$ topology. Write

$$
\gamma_{n}(\theta)=\sum_{k \in \mathbb{Z}} A_{k n} e^{i k \theta},
$$

where $A_{k n} \in M^{N \times N}(\mathbb{C})$ and only finitely many $A_{k n} \neq 0$ for each $n$. Fix $K$ a positive integer. Consider

$$
V:=\left\{\gamma \in L_{\mathrm{alg}}\left(M^{N \times N}(\mathbb{C})\right) \text { of type }(2-1):\left|A_{k}\right|<2^{-K-|k|} \forall k \in \mathbb{Z}\right\},
$$

which is open in the direct limit topology and contains the matrix 0 . The convergence of $\left(\gamma_{n}\right)$ to 0 in the direct limit topology implies that there exists a positive integer $n(K)$ such that for any $n \geq n(K)$ we have $\gamma_{n} \in V$, which means

$$
\left|A_{k n}\right|<2^{-K-|k|}, \forall k \in \mathbb{Z} \text {. }
$$

In order to prove the convergence in the $H^{1}$ topology, recall that

$$
\left\|\gamma_{n}\right\|_{H^{1}}:=\frac{1}{2 \pi} \int_{0}^{2 \pi}\left(\gamma_{n}(\theta), \gamma_{n}(\theta)\right)+\frac{1}{2 \pi} \int_{0}^{2 \pi}\left(\gamma_{n}^{\prime}(\theta), \gamma_{n}^{\prime}(\theta)\right)=\sum_{k \in \mathbb{Z}}\left(1+k^{2}\right)\left|A_{k n}\right|^{2},
$$

which is less than

$$
2^{-K} \sum_{k \in \mathbb{Z}} \frac{1+k^{2}}{2^{|k|}}
$$

Because $\sum_{k \in \mathbb{Z}} \frac{1+k^{2}}{2^{|k|}}$ is finite, this implies $\left\|\gamma_{n}\right\|_{H^{1}} \rightarrow 0$, and hence $\gamma_{n}$ converges to 0 in the $H^{1}$ topology as desired.

Remark In general, the direct limit topology on $\Omega_{\text {alg }}(G)$ is strictly finer than the subspace topology. For example, for $G=S U(2)$, we can consider the sequence $\gamma_{n} \in \Omega_{\mathrm{alg}}(S U(2))$ given by

$$
\gamma_{n}(z)=\left(\begin{array}{cc}
\sqrt{1-\frac{1}{n^{4}}} & \frac{1}{n^{2}} z^{n} \\
-\frac{1}{n^{2}} z^{-n} & \sqrt{1-\frac{1}{n^{4}}}
\end{array}\right)
$$

where $z=e^{i \theta}$. We can see that $\left(\gamma_{n}\right)$ converges to the constant loop $I_{2}$ in the $H^{1}$ topology, but not in the direct limit topology. To prove the first claim, we note that

$$
\left\|\gamma_{n}-I_{2}\right\|_{H_{1}}^{2}=\left\|\frac{1}{n^{2}} z^{-n}\right\|_{H_{1}}^{2}+2\left(1-\sqrt{1-\frac{1}{n^{4}}}\right)^{2}+\left\|\frac{1}{n^{2}} z^{n}\right\|_{H_{1}}^{2}
$$


which is convergent to 0 . In order to prove the nonconvergence of $\left(\gamma_{n}\right)$ in the direct limit topology, we write

$$
\gamma_{n}(z)=-\frac{1}{n^{2}}\left(\begin{array}{ll}
0 & 0 \\
1 & 0
\end{array}\right) z^{-n}+\left(\begin{array}{cc}
\sqrt{1-\frac{1}{n^{4}}} & 0 \\
0 & \sqrt{1-\frac{1}{n^{4}}}
\end{array}\right)+\frac{1}{n^{2}}\left(\begin{array}{ll}
0 & 1 \\
0 & 0
\end{array}\right) z^{n} .
$$

Let $U$ be the (open) subspace of $L_{\mathrm{alg}}\left(M^{2 \times 2}(\mathbb{C})\right)$ consisting of all series of the type (2-1) where $n$ is arbitrary and $\left|A_{k}\right|<2^{-|k|}$, for all $k \in \mathbb{Z}$. Suppose that $\left(\gamma_{n}\right)$ converges to $I_{2}$ in the direct limit topology. This implies that there exists $n_{0}$ such that for any $n \geq n_{0}$, we have $\gamma_{n} \in U$. This implies

$$
\left|\frac{1}{n^{2}}\left(\begin{array}{ll}
0 & 1 \\
0 & 0
\end{array}\right)\right|<2^{-n}
$$

for all $n \geq n_{0}$, which is false.

\subsection{The Grassmannian model}

The main reference here is [26], sections 7 and 8 (see also [2, Section 2]). Let $H$ be the Hilbert space $L^{2}\left(S^{1}, \mathbb{C}^{N}\right)$ and $H_{+}$the (closed) subspace of $H$ consisting of all elements with Fourier expansions of the type

$$
\sum_{k \geq 0} a_{k} e^{i k \theta}
$$

where $a_{k} \in \mathbb{C}^{N}$. Denote by $H_{-}$the orthogonal complement of $H_{+}$in $H$. The infinite-dimensional Grassmannian $\operatorname{Gr}(H)$ is the space of all closed linear subspaces $W \subset H$ such that

(i) the orthogonal projection $\mathrm{pr}_{+}: W \rightarrow H_{+}$is a Fredholm operator,

(ii) the orthogonal projection $\mathrm{pr}_{-}: W \rightarrow H_{-}$is a Hilbert-Schmidt operator.

This Grassmannian $\operatorname{Gr}(H)$ is a Hilbert manifold which has a Kähler form $\omega$ (see [26, Section 7.8]). We also identify the following important submanifolds of $\operatorname{Gr}(H)$, that we will use in the sequel. First, $G r_{\infty}(H)$ (called the smooth Grassmannian) is the space of all $W \in G r(H)$ for which the images of both orthogonal projections $W \rightarrow H_{-}$and $W^{\perp} \rightarrow H_{+}$consist of smooth functions. By $G r_{0}(H)$ one denotes the space of all $W \in G r(H)$ with the property that there exists an integer $n \geq 0$ such that

$$
e^{i n \theta} H_{+} \subset W \subset e^{-i n \theta} H_{+} .
$$

One can show that $G r_{0}(H) \subset G r_{\infty}(H)$ (see [26, Section 7.2]). 
Like in section 2.1, we assume that $G$ is a subgroup of $S U(N)$. The (linear) action of $T$ on $\mathbb{C}^{N}$ induces in a natural way actions on $H$ and on $\operatorname{Gr}(H)$. There also exists a "rotation" action of $S^{1}$ on $H$, given by

$$
\left(e^{i \theta} f\right)(z):=f\left(e^{i \theta} z\right),
$$

for all $e^{i \theta}, z \in S^{1}$ and all $f \in H$. It turns out that if $W \in G r(H)$, then the space

$$
e^{i \theta} W:=\left\{e^{i \theta} f: f \in W\right\}
$$

is also in $\operatorname{Gr}(H)$. The actions of $T$ and $S^{1}$ on $\operatorname{Gr}(H)$ commute with each other and induce an action of $T \times S^{1}$ on $G r(H)$. The space $G r_{\infty}(H)$ is $T \times S^{1}$ invariant (see [26, Section 7.6]). Now if $W \in G r(H)$, the map $S^{1} \rightarrow G r(H)$ given by $e^{i \theta} \mapsto e^{i \theta} W$ is in general not smooth. The latter map is smooth provided that $W$ is in the smooth Grassmannian $G r_{\infty}(H)$. In this way, we obtain a smooth action of $T \times S^{1}$ on $G r_{\infty}(H)$. This fits well with the symplectic structure on $\operatorname{Gr}(H)$, as indicated in the following proposition.

Proposition 2.2 [26, Proposition 7.8.2] There exists a function $\tilde{\mu}: G r_{\infty}(H) \rightarrow \mathfrak{t} \oplus \mathbb{R}$ such that for any $\xi \in \mathfrak{t} \oplus \mathbb{R}$, we have

$$
d\langle\tilde{\mu}, \xi\rangle=\omega\left(X_{\xi}, \cdot\right)
$$

where $X_{\xi}$ denotes the infinitesimal vector field on $G r_{\infty}(H)$ induced by $\xi$.

The space $G r_{0}(H)$ is also $T \times S^{1}$ invariant. Moreover, it is invariant under the natural action of the complexified torus $T^{\mathbb{C}}$ on $\operatorname{Gr}(H)$, which is induced by the action of $T^{\mathbb{C}}$ on $H$. Combined with the action of $\left(S^{1}\right)^{\mathbb{C}}=\mathbb{C}^{*}$ defined in [26, Section 7.6], this gives an action of the complex torus $T^{\mathbb{C}} \times \mathbb{C}^{*}$ on $G r_{0}(H)$, which extends the action of $T \times S^{1}$. Note that for a fixed $n$, the identification

$$
W \mapsto W / e^{i n \theta} H_{+} \subset e^{-i n \theta} H_{+} / e^{i n \theta} H_{+}=\mathbb{C}^{2 n N}
$$

makes the space of all $W$ that satisfy equation (2-2) into a finite-dimensional Grassmannian, denoted

$$
\mathcal{G}_{n}:=\operatorname{Gr}\left(n N, \mathbb{C}^{2 n N}\right)
$$

Thus we may think of $\mathcal{G}_{n}$ as a subset of $G r_{\infty}(H)$. By the definition of the action, it is straightforward to see that each $\mathcal{G}_{n} \subseteq G r_{\infty}(H)$ is $T^{\mathbb{C}} \times \mathbb{C}^{*}$-invariant. The following proposition summarizes the facts we need about the "Grassmannian model" [26, Section 8.3], [19, Lemma 2.4]. 
Proposition 2.3 (a) Let $\Omega_{\infty}(G)$ denote the space of all smooth loops $S^{1} \rightarrow G$. The map $\imath_{1}: \Omega_{\infty}(G) \rightarrow G r_{\infty}(H)$ given by $\iota_{1}(\gamma):=\gamma H_{+}$, is injective. We have $\left.\mu\right|_{\Omega_{\infty}(G)}=\tilde{\mu} \circ l_{1}$.

(b) We have $\Omega_{\mathrm{alg}}(G) \subset \Omega_{\infty}(G)$ and $\iota_{1}\left(\Omega_{\mathrm{alg}}(G)\right) \subset G r_{0}(H)$. Consequently, $\iota_{1}\left(\Omega_{\mathrm{alg}}(G)\right)$ is contained in $\bigcup_{n \geq 0} X_{n}$, where $X_{n}$ denotes the space of all closed linear subspaces $W \subset H$ with the properties

$$
e^{i n \theta} H_{+} \subset W \subset e^{-i n \theta} H_{+}, \quad e^{i \theta} W \subset W .
$$

(c) The map $W \mapsto W / e^{i n \theta} H_{+}$identifies $X_{n}$ with a subvariety of the Grassmannian $\mathcal{G}_{n}$ of all complex vector subspaces of $e^{-i n \theta} H_{+} / e^{i n \theta} H_{+} \cong \mathbb{C}^{2 n N}$ of dimension $n N$. The subspace $\mathcal{G}_{n} \hookrightarrow G r_{\infty}(H)$ is $T \times S^{1}$ invariant. The restriction of the Kähler form on $\operatorname{Gr}(H)$ to $\mathcal{G}_{n}$ is equal to the Kähler structure induced on $\mathcal{G}_{n} \cong \operatorname{Gr}\left(n N, \mathbb{C}^{2 n N}\right)$ via the Plücker embedding into $\mathbb{P}^{M}$, where $M=\left(\begin{array}{c}2 n N \\ n N\end{array}\right)$.

\subsection{Morse theory for the components of $\mu$}

In this subsection we discuss Morse theory for the components of the moment map $\mu: \Omega_{1}(G) \rightarrow \mathfrak{t} \oplus \mathbb{R}$. We recall that an element of $\mathfrak{t}$ is called regular if it is not contained in any of the hyperplanes $\operatorname{ker} \alpha \subseteq \mathfrak{t}$, where $\alpha \in \mathfrak{t}^{*}$ is a root of $G .^{1}$ The connected components of $\mathfrak{t} \backslash \bigcup_{\alpha \text { root }}$ ker $\alpha$ are called Weyl chambers. We will need the following result (for a proof, one can see for instance [3, Chapter V, Proposition 2.3]).

Lemma 2.4 If $X$ is an element of $\mathfrak{t}$ then the following two assertions are equivalent.

(i) The centralizer of $\exp (X)$ reduces to $T$.

(ii) The vector $X$ does not belong to any of the affine hyperplanes

$$
H_{\alpha, k}:=\{H \in \mathfrak{t} \mid \alpha(H)=k\},
$$

where $\alpha$ is a root and $k \in \mathbb{Z}$.

If $X \in \mathfrak{t}$ is an arbitrary regular vector, then there exists a positive integer $q(X)$ such that for any $q \geq q(X)$, the vector $X / q$ satisfies condition (ii) from above.

Definition 2.5 An element $\rho \in \mathfrak{t}$ is called admissible if it is of the type $X / q$, where $X$ is a regular element which belongs to the integer lattice and $q \geq q(X)$.

\footnotetext{
${ }^{1}$ We apologize to the reader for the confusing terminology; a "regular element of $t$ " means something different from a "regular value of the moment map." Unfortunately both terms are fairly standard in their respective contexts, so we use both here. We hope the context makes clear which we mean.
} 
We define a pairing on the Lie algebra $\mathfrak{t} \oplus \mathbb{R}$ by taking the restriction of the pairing on $\mathfrak{g}$ on the first factor, taking the standard inner product on the second factor, and declaring the two factors to be orthogonal. We denote this pairing also by $\langle\cdot, \cdot\rangle$. For the purposes of the Morse theoretical arguments in the following sections, we are interested in the functions $\langle\mu(\cdot),(0,1)\rangle$ and $\langle\mu(\cdot),(\rho, 1)\rangle$, where $\rho \in \mathfrak{t}$ is an admissible element. The first function is the energy functional $E$, namely the $S^{1}$-component of the moment map. The second one is, up to an additive constant $c=c(\rho)$, the "tilted" energy $\widetilde{\mathcal{E}}[26$, Section 8.9], ie,

$$
\langle\mu(\gamma),(\rho, 1)\rangle=c+\frac{1}{4 \pi} \int_{0}^{2 \pi}\left\|\gamma(\theta)^{-1} \gamma^{\prime}(\theta)+\rho\right\|^{2} d \theta,
$$

$\gamma \in \Omega_{1}(G)$. Both of these functions have a good corresponding Morse theory with respect to the Kähler metric on $\Omega_{1}(G)$; in particular, the downward gradient flow with respect to both of these functions is well-defined for all time $t$, and both the critical sets and their unstable manifolds have explicit descriptions. The following two results are proved in [26, Section 8.9] (see also [15, Section 3.1]). We denote by $\nabla f$ the gradient vector field corresponding to a function $f$.

Proposition 2.6 (a) Let $\rho$ be admissible vector in $\mathfrak{t}$. The critical set of $\mu^{(\rho, 1)}:=$ $\langle\mu(\cdot),(\rho, 1)\rangle$ is the lattice $\check{T}$ of group homomorphisms $\lambda: S^{1} \rightarrow T$. If $\gamma \in$ $\Omega_{\mathrm{alg}}(G)$, then the solution $\phi_{t}(\gamma)$ of the initial value problem

$$
\frac{d}{d t} \phi_{t}(\gamma)=-\nabla\left(\mu^{(\rho, 1)}\right)_{\phi_{t}(\gamma)}, \quad \phi_{0}(\gamma)=\gamma
$$

is defined for all $t \in \mathbb{R}$. Moreover, $\phi_{t}(\gamma) \in \Omega_{\mathrm{alg}}(G)$ for all $t \in \mathbb{R}$, the limit $\lim _{t \rightarrow-\infty} \phi_{t}(\gamma)$ exists, and it is a critical point.

(b) If $\lambda$ is a critical point of $\mu^{(\rho, 1)}$, then the unstable manifold

$$
C_{\lambda}(\rho):=\left\{\gamma \in \Omega_{\text {alg }}: \lim _{t \rightarrow-\infty} \phi_{t}(\gamma)=\lambda\right\}
$$

is homeomorphic to $\mathbb{C}^{m(\lambda, \rho)}$, for a certain number $m(\lambda, \rho)$.

(c) We have the cell decomposition

$$
\Omega_{\mathrm{alg}}(G)=\bigcup_{\lambda \in \check{T}} C_{\lambda}(\rho) .
$$

(d) For any $\rho$ in the positive Weyl chamber, the $C_{\lambda}(\rho)$ are the Bruhat cells $C_{\lambda}$ (as in [26, Section 8.6]).

We have a similar result for the other component of $\mu$, namely $E$. The main difference is that the critical sets are no longer isolated, but instead are diffeomorphic to coadjoint orbits of $G$. 
Proposition 2.7 (a) The critical set of the energy functional $E=\langle\mu(\cdot),(0,1)\rangle$ is the union of the spaces

$$
\Lambda_{|\lambda|}=\left\{g \lambda g^{-1}: g \in G\right\},
$$

where $|\lambda| \in \check{T} / W$. If $\gamma \in \Omega_{\mathrm{alg}}(G)$, then the solution $\psi_{t}(\gamma)$ of the initial value problem

$$
\frac{d}{d t} \psi_{t}(\gamma)=-\nabla E_{\psi_{t}(\gamma)}, \quad \psi_{0}(\gamma)=\gamma
$$

is defined for all $t \in \mathbb{R}$. Moreover, $\psi_{t}(\gamma) \in \Omega_{\text {alg }}(G)$ for all $t \in \mathbb{R}$, the limit $\lim _{t \rightarrow-\infty} \psi_{t}(\gamma)$ exists, and it is a critical point.

(b) For any $\lambda \in \check{T}$, the unstable manifold

$$
C_{|\lambda|}:=\left\{\gamma \in \Omega_{\mathrm{alg}}: \lim _{t \rightarrow-\infty} \phi_{t}(\gamma) \in \Lambda_{|\lambda|}\right\}
$$

is a closed submanifold of $\Omega_{1}(G)$.

(c) We have the decomposition

$$
\Omega_{\mathrm{alg}}(G)=\bigcup_{\lambda \in \check{T} / W} C_{|\lambda|} .
$$

It is known that many results of Morse theory remain true for real functions on infinite dimensional Riemannian manifolds, provided that they satisfy the condition (C) of Palais and Smale. The latter condition is as follows (see for instance [24, Chapter 9]).

Definition 2.8 (Condition (C)) We say that a real function $f$ on a Riemannian manifold $M$ satisfies the condition (C) if any sequence $\{s(n)\}$ of points in $M$ for which $\left\{(f(s(n))\}\right.$ is bounded and $\lim _{n \rightarrow \infty}\left\|\nabla(f)_{s(n)}\right\|=0$ has a convergent subsequence.

It turns out that the components of the moment map on $\Omega_{1}(G)$ do satisfy this condition, with respect to the $H^{1}$ metric (see Section 2.1). Indeed, let us consider the "gauge" action of $L_{1}(G)=H^{1}\left(S^{1}, G\right)$ on $H^{0}\left(S^{1}, \mathfrak{g}\right)$, given by

$$
\gamma \star u=\gamma u \gamma^{-1}-\gamma^{\prime} \gamma^{-1}
$$

for all $\gamma \in L_{1}(G)$ and all $u \in H^{0}\left(S^{1}, \mathfrak{g}\right)$. This action is proper and Fredholm, which implies that if we regard any of its orbits as a sumbanifold of (the Hilbert space) $H^{0}\left(S^{1}, \mathfrak{g}\right)$, then for any $a \in H^{0}\left(S^{1}, g\right)$ the distance function $f_{a}(x)=\|x+a\|^{2}$, with $x$ on the orbit, satisfy the condition (C) (see [27, Proposition 2.16 and Section 4]). The stabilizer of the constant loop $0 \in H^{0}\left(S^{1}, \mathfrak{g}\right)$ consists of all constant loops in $G$, thus the orbit of 0 is $L_{1}(G) / G=\Omega_{1}(G)$. It is not difficult to see that any element of the orbit can be written uniquely as $\gamma^{-1} \gamma^{\prime}$, with $\gamma \in \Omega_{1}(G)$. It turns out (see again [27, 
Section 4]) that the metric on $\Omega_{1}(G)$ induced by its embedding in $H^{0}\left(S^{1}, \mathfrak{g}\right)$ is just the $H^{1}$ metric. We summarize as follows.

Proposition 2.9 Regard $\Omega_{1}(G)$ as a Riemannian manifold with respect to the $H^{1}$ metric. The map

$$
F: \Omega_{1}(G) \rightarrow H^{0}\left(S^{1}, \mathfrak{g}\right), \quad F(\gamma)=\gamma^{-1} \gamma^{\prime}
$$

is an isometric embedding. For any $\rho \in \mathfrak{t}$, the function $f_{\rho}: F\left(\Omega_{1}(G)\right) \rightarrow \mathbb{R}$ given by $f_{\rho}(x)=\|x+\rho\|^{2}$ satisfies the condition $(C)$. Consequently, the function $\langle\mu,(\rho, 1)\rangle=$ $c+\frac{1}{4 \pi} f_{\rho} \circ F$ (see equation (2-4)) defined on $\Omega_{1}(G)$ satisfies the condition $(C)$ as well.

The fact that the components of the moment map $\mu$ on $\Omega_{1}(G)$ satisfy condition (C) will be the key element in our arguments in Sections 4 and 5 .

\subsection{Geometric invariant theory}

In our proof of the connectivity result for the algebraic loops $\Omega_{\mathrm{alg}}(G)$, we will heavily use the fact that in certain cases, the symplectic quotient is the same as the geometric invariant theory quotient (in the sense of algebraic geometry). The main reference for this section is the book [22] (see also [13; 14; 4]). Assume that a complex torus $T^{\mathbb{C}}=\left(\mathbb{C}^{*}\right)^{k}$ acts analytically on a complex projective irreducible (possibly singular) variety $X$. Let $L$ be an ample $T^{\mathbb{C}}$-line bundle on $X$. A point $x \in X$ is called $L$-semistable if there exists $m \geq 1$ and a section $s$ of the tensor bundle $L^{\otimes m}$ with $s(x) \neq 0$. The set $X^{s s}(L)$ of all semistable points in $X$ is a Zariski open, hence irreducible, subvariety of $X$. If $Z \subset X$ is a subvariety, then

$$
Z^{s s}(L)=Z \cap X^{s s}(L) .
$$

Now suppose that $Y \subset \mathbb{P}^{M}$ is a smooth projective variety, invariant under the linear action of $T^{\mathbb{C}}=\left(\mathbb{C}^{*}\right)^{k}$ on $\mathbb{P}^{M}$. Take $\mu: Y \rightarrow \mathfrak{t}:=\operatorname{Lie}(T)$ to be the restriction to $Y$ of any $T$-moment map on $\mathbb{P}^{M}$. We say that $x \in Y$ is $\mu$-semistable if $\overline{T^{\mathbb{C}} . x} \cap \mu^{-1}(0) \neq \phi$. We denote by $Y^{s s}(\mu)$ the set of all $\mu$-semistable points in $Y$. The following result is Theorem 8.10 in [13]:

Theorem 2.10 Let $Y^{\mathrm{min}}$ denote the minimal Morse-Kirwan stratum of $Y$ with respect to the function $f=\|\mu\|^{2}$, corresponding to the critical set $f^{-1}(0)=\mu^{-1}(0)$. Then $Y^{s s}(\mu)=Y^{\mathrm{min}}$.

In order to relate the $L$-semistable points and $\mu$-semistable points, we will also need the following result of Heinzner and Migliorini [10]. 
Theorem 2.11 [10] There exists an ample $T^{\mathbb{C}}$-line bundle $L$ such that $Y^{s s}(L)=$ $Y^{s s}(\mu)$.

\section{The levels of moment maps on algebraic loops}

The goal of this section is to prove that all non-empty levels of both $E: \Omega_{\mathrm{alg}}(G) \rightarrow \mathbb{R}$ and $\mu: \Omega_{\text {alg }}(G) \rightarrow \mathfrak{t} \oplus \mathbb{R}$ are connected. The essential idea of the proof is to use the decomposition of $\Omega_{\mathrm{alg}}(G)$ into Bruhat cells, given in Proposition 2.6. We will first prove that the level sets on the closure of each Bruhat cell $C_{\lambda}$ are connected, using the fact that each such closure $\overline{C_{\lambda}}$ can be interpreted as a $T$-invariant subvariety of an appropriate projective space. This allows us to use geometric invariant theory results (Theorems 2.10 and 2.11) to prove the connectivity result on each $\overline{C_{\lambda}}$. We then use the closure relations on the Bruhat cells to prove that the connectivity result for each cell is sufficient to show that the level set on the union $\Omega_{\mathrm{alg}}(G)=\cup_{\lambda} C_{\lambda}$ is also connected.

In order to use the results outlined in Section 2.4, we must first prove that the spaces under consideration are indeed $T$-invariant Zariski-closed subvarieties of an appropriate projective space. Recall that $\Omega_{\mathrm{alg}}(G)$ contains subvarieties of finite-dimensional Grassmannians $\mathcal{G}_{n}$, as explained in Section 2.2. On the other hand, the Grassmannians $\mathcal{G}_{n}=\operatorname{Gr}\left(n, \mathbb{C}^{2 n}\right)$ may also be seen as a subvariety of a projective space via the Plücker embedding. We first observe that this Plücker embedding is compatible with the torus action on $\Omega_{\text {alg }}(G)$.

Lemma 3.1 Let $\varphi: \mathcal{G}_{n} \hookrightarrow \mathbb{P}^{M}$ be the Plücker embedding of the Grassmannian $\mathcal{G}_{n}$ where $M:=\left(\begin{array}{c}2 n N \\ n N\end{array}\right)$. Then the action of $T^{\mathbb{C}} \times \mathbb{C}^{*}$ on $\mathcal{G}_{n}$ extends to a linear action on $\mathbb{p}^{M}$.

Proof By definition, $\mathcal{G}_{n}$ is the space of all $n N$-dimensional subspaces of

$$
e^{-i n \theta} H_{+} / e^{i n \theta} H_{+} \simeq\left\langle e^{-i k \theta} b_{j}:-n \leq k \leq n-1,1 \leq j \leq N\right\rangle \simeq \mathbb{C}^{2 k n},
$$

where $b_{1}, \ldots, b_{N}$ is the canonical basis of $\mathbb{C}^{N}$. Recall that in order to define the Plücker embedding, we specify an element of the $\mathcal{G}_{n}$ by a $2 n N \times n N$ matrix $A$ of rank $n N$; such a matrix specifies a subspace of $\mathbb{C}^{2 N}$ by taking the span of its columns. The Plücker embedding then maps $A$ to $\mathbb{P}^{M}$ by taking the coordinates in $\mathbb{P}^{M}$ to be the $n N \times n N$ minors of $A$. This is well-defined.

We wish to show that this standard Plücker embedding is $T^{\mathbb{C}} \times \mathbb{C}^{*}$-equivariant, and in fact the action extends to a linear $T^{\mathbb{C}} \times \mathbb{C}^{*}$-action on $\mathbb{P}^{M}$. By our assumption on $T$ (see Section 2.2), $T^{\mathbb{C}}$ acts on $\mathbb{C}^{N}$ as left multiplication by diagonal matrices. 
The corresponding action of $T^{\mathbb{C}}$ on the matrix $A$, which represents an element in $\mathcal{G}_{n}$, is therefore also given by left multiplication by a diagonal matrix. It is then straightforward to check that the action of $T^{\mathbb{C}}$ extends to a linear (indeed, diagonal) action on $\mathbb{P}^{M}$.

It remains to show that the extra $\mathbb{C}^{*}$-action also extends linearly to $\mathbb{P}^{M}$. Since this action is to "rotate the loop," an element $z$ of $\mathbb{C}^{*}$ acts as follows:

$$
z \cdot\left(e^{i k \theta} b_{j}\right)=\left(z e^{i \theta}\right)^{k} b_{j}=z^{k}\left(e^{i k \theta} b_{j}\right),
$$

$-n \leq k \leq n-1,1 \leq j \leq N$. Again, regarded as a linear transformation of $\mathbb{C}^{2 n N}, z$ is diagonal. We use the same argument as before to complete the proof.

We are now prepared to prove the connectivity for each Bruhat cell. Let $\lambda \in \check{T}$. There exists $n \geq 1$ such that $\iota_{1}\left(\overline{C_{\lambda}}\right) \subset X_{n}$ (see eg, [2]). Here $\overline{C_{\lambda}}$ represents the closure of $C_{\lambda}$ in $\Omega_{\mathrm{alg}}(G)$ in the direct limit topology, as explained in the Introduction. The $T \times S^{1}$-equivariance of the Plücker embedding in the previous lemma allows us to analyze the level set of the moment map $\mu$ on $\overline{C_{\lambda}}$ as that of a moment map defined on a finite-dimensional space, namely the Grassmannian. The next result is a direct consequence of Proposition 2.3 (see also [19, Lemma 2.4]).

Lemma 3.2 The following diagram is commutative.

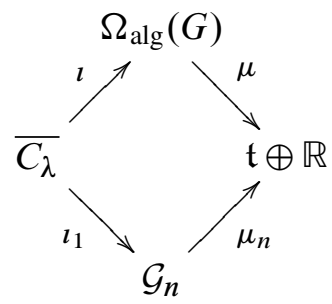

Here $l: \overline{C_{\lambda}} \rightarrow \Omega_{\text {alg }}(G)$ denotes the inclusion map, and $\mu_{n}: \mathcal{G}_{n} \rightarrow \mathfrak{t} \oplus \mathbb{R}$ is the moment map corresponding to the action of $T \times S^{1}$ on $\mathcal{G}_{n}$ and the symplectic form on $\mathcal{G}_{n}$ induced by the Plücker embedding $\varphi: \mathcal{G}_{n} \hookrightarrow \mathbb{P}^{M}$.

Proof By Proposition 2.3 (a), we have

$$
\mu \circ \imath=\left.\mu\right|_{\overline{C_{\lambda}}}=\left.\tilde{\mu} \circ l_{1}\right|_{\overline{C_{\lambda}}} .
$$

We only need to show that $\left.\tilde{\mu}\right|_{\mathcal{G}_{n}}=\mu_{n}$. We have already seen in Proposition 2.3 that the symplectic structures on $\mathcal{G}_{n}$ coming from the inclusions $\mathcal{G}_{n} \hookrightarrow \mathbb{P}^{M}$ and $\mathcal{G}_{n} \hookrightarrow \operatorname{Gr}(H)$ are equal. Hence it is sufficient to see that the two inclusions are $T \times S^{1}$-equivariant. For the first inclusion, this statement is the content of Lemma 3.1, and the latter was observed in Section 2.2 (see Proposition 2.3 (c)). 
We can now prove the connectivity result on each closed Bruhat cell, using the geometric invariant results of Section 2.4.

Lemma 3.3 If $\lambda \in \check{T}$, then for any value $a \in \mathfrak{t} \oplus \mathbb{R}$, the level set $\mu^{-1}(a) \cap \overline{C_{\lambda}}$ is either empty or path connected.

Proof By Lemma 3.2, we have

$$
\mu^{-1}(a) \cap \overline{C_{\lambda}}=\mu_{n}^{-1}(a) \cap \overline{C_{\lambda}} .
$$

Hence it suffices to prove that the RHS is connected. We consider the function $f: \mathcal{G}_{n} \rightarrow$ $\mathbb{R}, f(x)=\left\|\mu_{n}(x)-a\right\|^{2}$, as well as its gradient flow $\Phi_{t}$. We will prove first that $\Phi_{t}$ leaves $\overline{C_{\lambda}}$ invariant. It suffices to show that the gradient flow of $f$ leaves any $T^{\mathbb{C}} \times \mathbb{C}^{*}$ orbit in $\mathcal{G}_{n}$ invariant, since $C_{\lambda}$ is $T^{\mathbb{C}} \times \mathbb{C}^{*}$-invariant. Recall that the gradient vector field of $f$ is given by

$$
\operatorname{grad}(f)_{x}=J\left(\mu_{n}(x)-a\right) \cdot x
$$

where the dot denotes the infinitesimal action of $\operatorname{Lie}\left(T \times S^{1}\right)=\mathfrak{t} \oplus \mathbb{R}$ on $\mathcal{G}_{n}$ at the point $x \in \mathcal{G}_{n}$, and $J$ denotes the complex structure. We deduce that the vector field $\operatorname{grad}(f)$ is tangent to a $T^{\mathbb{C}} \times \mathbb{C}^{*}$ orbit, hence any integral curve of $\operatorname{grad}(f)$ remains in an orbit.

Recall that the function $f$ induces a stratification of $\mathcal{G}_{n}$ ([13]) as follows. Let $\left\{C_{j}\right\}_{j=0}^{\ell}$ be the critical sets of $f$ in $\mathcal{G}_{n}$, ordered by their values $f\left(C_{j}\right)$, where $C_{0}=f^{-1}(0)=$ $\mu_{n}^{-1}(a)$ is the minimum. To each $C_{j}$ corresponds the stratum

$$
S_{j}=\left\{x \in \mathcal{G}_{n}: \lim _{t \rightarrow \infty} \Phi_{t}(x) \in C_{j}\right\} .
$$

Each $S_{j}$ is a complex submanifold of $\mathcal{G}_{n}$, which contains $C_{j}$ as a deformation retract $[30 ; 18 ; 22]$. The unique open stratum $S_{0}$ coincides with the set $\left(\mathcal{G}_{n}\right)^{s s}\left(\mu_{n}-a\right)$ of all semi-stable points in $\mathcal{G}_{n}$ (Theorem 2.10). By Theorem 2.11, there exists an ample $T^{\mathbb{C}} \times \mathbb{C}^{*}$-line bundle $L$ such that

$$
\left(\mathcal{G}_{n}\right)^{S s}\left(\mu_{n}-a\right)=\left(\mathcal{G}_{n}\right)^{s s}(L) .
$$

Since $\overline{C_{\lambda}}$ is a subvariety of $\mathcal{G}_{n}$, the set of all semi-stable points of $\overline{C_{\lambda}}$ is

$$
\left(\overline{C_{\lambda}}\right)^{s s}(L)=\left(\mathcal{G}_{n}\right)^{s s}(L) \cap \overline{C_{\lambda}}=S_{0} \cap \overline{C_{\lambda}} .
$$

Since $\overline{C_{\lambda}}$ is irreducible (see eg, [21]), $\left(\overline{C_{\lambda}}\right)^{s s}(L)$ is irreducible as well. Since any irreducible complex analytic projective variety has a resolution of singularities, $\left(\overline{C_{\lambda}}\right)^{s s}(L)$ is path-connected in the usual differential topology of $\mathbb{P}^{M}$. We deduce that $S_{0} \cap \overline{C_{\lambda}}$ is path-connected. Because $\overline{C_{\lambda}}$ is invariant under $\Phi_{t}$, the space $S_{0} \cap \overline{C_{\lambda}}$ contains $C_{0} \cap \overline{C_{\lambda}}=\mu_{n}^{-1}(a) \cap \overline{C_{\lambda}}$ as a deformation retract. Thus the latter is path-connected as well. 
We now use Lemma 3.3 and the closure relations for the Bruhat cells to prove the connectivity result for all of $\Omega_{\mathrm{alg}}(G)$.

Proposition 3.4 For any $a \in \mathfrak{t} \oplus \mathbb{R}$, the set $\mu^{-1}(a) \cap \Omega_{\mathrm{alg}}(G)$ is empty or connected.

Proof Let $\gamma_{1}$ and $\gamma_{2}$ be in $\mu^{-1}(a) \cap \Omega_{\mathrm{alg}}(G)$. Since the union of the Bruhat cells equals $\Omega_{\text {alg }}(G)$, there exist $\lambda_{1}, \lambda_{2}$ in the integer lattice $\check{T}$ such that $\gamma_{1} \in C_{\lambda_{1}}, \gamma_{2} \in C_{\lambda_{2}}$. Let us consider the ordering $\leq$ on $\check{T}$ given by $\lambda \leq v$ if and only if $C_{\lambda} \subset \overline{C_{\nu}}$. One can show that this is the same as the Bruhat ordering on $\check{T}$ (see for instance [21, Theorem 1.3]). This is induced (see [16, Section 1.3]) by the Bruhat ordering on the affine Weyl group $W_{\text {aff }}=\check{T} \rtimes W$, which is a Coxeter group, via the obvious identification $\check{T}=W_{\text {aff }} / W$. One of the properties of the Bruhat ordering on Coxeter groups is that for any two elements there exists a third one which is "larger" than both of the previous two (see for instance [16, Lemma 1.3.20]). In our context this implies that there exists $v \in \check{T}$ such that $\lambda_{1} \leq v$ and $\lambda_{2} \leq v$, that is, $C_{\lambda_{1}} \subseteq \overline{C_{\nu}}$ and $C_{\lambda_{2}} \subseteq \overline{C_{\nu}}$. We apply Lemma 3.3 and deduce that $\mu^{-1}(a) \cap \Omega_{\mathrm{alg}}(G)$ is path connected, hence connected.

By using exactly the same methods, we can prove the following proposition.

Proposition 3.5 For any $a \in \mathbb{R}$, the energy level set $E^{-1}(a) \cap \Omega_{\mathrm{alg}}(G)$ is either empty or connected.

\section{The regular levels of the moment map on $\Omega(G)$}

In this section we prove the connectivity result for $\Omega_{1}(G)$, using the topology induced from $H^{1}$. By Proposition 3.4, we know that $\mu^{-1}(a) \cap \Omega_{\text {alg }}$ is a connected subspace of $\Omega_{\text {alg }}(G)$ in the direct limit topology. As observed in Section 1, the direct limit topology is strictly finer than the topology induced from $\Omega_{1}(G)$, so $\mu^{-1}(a) \cap \Omega_{\text {alg }}(G)$ is also connected in the $H^{1}$ topology. For the rest of the section, we consider only the $H^{1}$ topology.

In order to prove the connectivity result for $\Omega_{1}(G)$, we will heavily use the connectivity result for $\Omega_{\mathrm{alg}}(G)$, proven in the previous section. We also use that $\Omega_{\mathrm{alg}}(G)$ is dense in $\Omega_{1}(G)$ in the $H^{1}$ topology [2, Theorem 2]. Since the closure of a connected subset is also connected, in order to prove Theorem 1.2, it therefore suffices to prove the following proposition.

Proposition 4.1 If $a \in \mathfrak{t} \oplus \mathbb{R}$ is a regular value of $\mu$, then $\mu^{-1}(a) \cap \Omega_{\mathrm{alg}}(G)$ is dense in $\mu^{-1}(a)$ in the $H^{1}$ topology. 
Remark 4.2 The reader may notice that the above proposition has an additional hypothesis of regularity of the value, which was not necessary for the case of $\Omega_{\mathrm{alg}}(G)$. We need this hypothesis to use the Morse-theoretic arguments below. We are not aware of other methods to prove Theorem 1.2, although the question of singular values is still of interest. We address the case of singular values for $E$ in the next section.

We now concentrate on the proof of Proposition 4.1. Before we proceed, we warn the reader that throughout the rest of this section, the loops will be generically denoted by $x$, rather than $\gamma$. In order to prove the density, we will construct for any point $x_{0} \in \mu^{-1}(a)$ a sequence contained in $\mu^{-1}(a) \cap \Omega_{\mathrm{alg}}(G)$ converging to it. We accomplish this by first using the fact that $\Omega_{\mathrm{alg}}(G)$ is dense in $\Omega_{1}(G)$ to find a sequence $x(r) \rightarrow x_{0} \in \mu^{-1}(a)$, where $x(r) \in \Omega_{\mathrm{alg}}(G)$. We then use the Morse flow with respect to independent components of the moment map $\mu$, restricted to $\Omega_{\mathrm{alg}}(G)$, to produce a new sequence $y(r) \rightarrow x_{0}$, where now $y(r) \in \mu^{-1}(a) \cap \Omega_{\mathrm{alg}}(G)$. It is precisely this construction which uses Morse flows with respect to components of the moment map $\mu$ - where we need the Palais-Smale condition (C).

We will need the following.

Lemma 4.3 Let $a$ be a regular value of $\mu: \Omega_{1}(G) \rightarrow \mathfrak{t} \oplus \mathbb{R}$, and let $x \in \mu^{-1}(a)$.

(a) The map $P: \mathfrak{t} \oplus \mathbb{R} \rightarrow T_{x}\left(\Omega_{1}(G)\right), \xi \mapsto \nabla\langle\mu(\cdot), \xi\rangle(x)$ is $\mathbb{R}$-linear and injective. Denote by $V:=P(\mathfrak{t} \oplus \mathbb{R}) \subset T_{x}\left(\Omega_{1}(G)\right)$ the image of this map.

(b) Denote $k-1:=\operatorname{dim}(\mathfrak{t})$. There exists a basis $\xi_{1}, \ldots, \xi_{k}$ of $\mathfrak{t} \oplus \mathbb{R}$ such that $\xi_{1}=$ $(0,1), \xi_{j}=\left(\rho_{j}, 1\right)$, where $\rho_{2}, \ldots, \rho_{k}$ are admissible (in the sense of Definition 2.5) elements of $\mathfrak{t}$ with the following property: let $v_{i}:=\nabla\left\langle\mu(\cdot), \xi_{i}\right\rangle(x)$, and $p_{i}: V \rightarrow V$ the orthogonal projection on the hyperplane orthogonal to $v_{i}$. Then

$$
g\left(v_{j}, p_{1} \circ \ldots \circ p_{j-1}\left(v_{j}\right)\right) \neq 0,
$$

for all $j \geq 2$. Here $g$ denotes the Kähler metric on $\Omega_{1}(G)$.

Proof We first prove claim (a). Since $a$ is a regular value, $T \times S^{1}$ acts locally freely on the level $\mu^{-1}(a)$, hence the linear map $\xi \mapsto \xi_{x}$ from $\mathfrak{t} \oplus \mathbb{R} \rightarrow T_{x} \Omega_{1}(G)$ is injective. The vector $\xi_{x}$ is the Hamiltonian vector field of $\langle\mu(\cdot), \xi\rangle$ at $x$. By properties of Hamiltonian vector fields on a Kähler manifold, $J_{x} \xi_{x}=\nabla\langle\mu(\cdot), \xi\rangle$, where $J_{x}$ denotes the complex structure at $x$. We then have that $P(\xi)=J_{x} \xi_{x}$, and since $J_{x}$ is a linear isomorphism at $T_{x} M$, the claim follows.

We now prove claim (b). We identify $V$ with $\mathfrak{t} \oplus \mathbb{R}$. This gives the identifications

$$
v_{1}:=P\left(\xi_{1}\right)=\xi_{1}:=(0,1), \quad \text { and } \quad v_{j}:=P\left(\xi_{j}\right)=\xi_{j}:=\left(\rho_{j}, 1\right), \quad 2 \leq j \leq k .
$$


The space $\mathfrak{t} \oplus \mathbb{R}$ inherits from $T_{x_{0}}(\Omega(G))$ an inner product via the map $P$. By abuse of notation we denote this metric on $\mathfrak{t} \oplus \mathbb{R}$ also by $g$. The argument is more easily given on $\mathfrak{t} \oplus \mathbb{R}$, but since the metric $g$ is the pullback from $T_{x} \Omega_{1}(G)$, the claim follows from the following construction of the $\rho_{i}$.

We inductively construct admissible vectors $\rho_{2}, \ldots, \rho_{k} \in \mathfrak{t}$ with the property that for any $\ell \geq 2$ we have

(i) the vectors $(0,1),\left(\rho_{2}, 1\right), \ldots,\left(\rho_{\ell}, 1\right)$ are linearly independent,

(ii) $g\left(v_{\ell}, p_{1} \circ \cdots \circ p_{\ell-1}\left(v_{\ell}\right)\right) \neq 0$,

(iii) $g\left((0,1),\left(\rho_{\ell}, 1\right)\right) \neq 0$.

Let $2 \leq \ell \leq k-1$. If $\ell \geq 3$ we assume that $\left\{\rho_{2}, \ldots, \rho_{\ell-1}\right\}$ have already been constructed. Now consider the function $f: \mathfrak{t} \rightarrow \mathbb{R}$, given by

$$
\begin{aligned}
f(\rho) & =g\left((\rho, 1), p_{1} \circ \cdots \circ p_{\ell-1}(\rho, 1)\right) \\
& =g\left((\rho, 0), p_{1} \circ \cdots \circ p_{\ell-1}(\rho, 1)\right) \\
& =g\left((\rho, 0), p_{1} \circ \cdots \circ p_{\ell-1}(0,1)\right)+g\left((\rho, 0), p_{1} \circ \cdots \circ p_{\ell-1}(\rho, 0)\right) .
\end{aligned}
$$

The two components of $f$ described by the previous equation are homogeneous polynomial functions of degree 1 , respectively 2 , on $\mathfrak{t}$.

We will prove that $f$ is not identically zero. To this end, it is sufficient to prove that the degree 1 component of $f$ is not identically zero. More specifically, we will prove the following claim.

Claim The function $\rho \mapsto g\left((\rho, 0), p_{1} \circ \cdots \circ p_{\ell-1}(0,1)\right), \rho \in \mathfrak{t}$ is not identically zero.

Proof of the claim First we show that $p_{1} \circ \ldots \circ p_{\ell-1}(0,1)=p_{1} \circ \ldots \circ p_{\ell-1}\left(\xi_{1}\right)$ is different from the 0 vector. To do that, we take into account that

$$
p_{j}(\xi)=\xi-\frac{g\left(\xi, \xi_{j}\right)}{g\left(\xi_{j}, \xi_{j}\right)} \xi_{j}
$$

where $1 \leq j \leq \ell-1$ and $\xi \in \mathfrak{t} \oplus \mathbb{R}$. From here, a straightforward computation that involves applying a projection of the form (4-1) successively $\ell-2$ times shows that $p_{2} \ldots p_{\ell-1}\left(\xi_{1}\right)$ is a linear combination of $\xi_{1}, \ldots, \xi_{\ell-1}$, where the coefficient of $\xi_{\ell-1}$ is $\frac{g\left(\xi_{1}, \xi_{\ell-1}\right)}{g\left(\xi_{\ell-1}, \xi_{\ell-1}\right)}$. The latter number is nonzero, by the induction hypothesis, hence $p_{2} \ldots p_{\ell-1}\left(\xi_{1}\right)$ cannot be collinear to $\xi_{1}$. This implies that $p_{1} \ldots p_{\ell-1}\left(\xi_{1}\right) \neq 0$, as stated above. Now if the function mentioned in the claim was identically 0 , from the fact that

$$
g\left((0,1), p_{1} \ldots p_{\ell-1}(0,1)\right)=0
$$


and the nondegeneracy of the metric $g$ on $\mathfrak{t} \oplus \mathbb{R}$, we deduce $p_{1} \ldots p_{\ell-1}(0,1)=0$, which is false. The claim is now proved.

We now note that condition (ii) is equivalent to the statement $f\left(\rho_{\ell}\right) \neq 0$. Similarly, conditions (i) and (iii) can be phrased as the non-vanishing of certain non-zero polynomials $f_{1}$ and $f_{3}$ on $\mathfrak{t}$. In particular, condition (i) is equivalent to the non-vanishing of all the $\ell \times \ell$ minors (and hence of their product, which we call $f_{1}$ ) of the matrix formed by the coordinates of the vectors $(0,1),\left(\rho_{2}, 1\right), \ldots,\left(\rho_{\ell}, 1\right)$. Similarly, condition (iii) is equivalent to the non-vanishing of the degree 1 polynomial $f_{3}(\rho):=g((0,1),(\rho, 1))$. Hence conditions (i), (ii), and (iii) are satisfied by any $\rho_{\ell}$ for which $f_{0}\left(\rho_{\ell+1}\right) \neq 0$, where we define

$$
f_{0}(\rho):=f_{1}(\rho) f(\rho) f_{3}(\rho) .
$$

Since $f$ was shown above to be not identically zero, and $f_{1}, f_{3}$ are also both not identically zero, $f_{0}$ is also not identically zero.

Let $\Lambda$ denote the integer lattice in $\mathfrak{t}$. Since a non-zero polynomial cannot vanish on all points of a full lattice, there exists $X \in \Lambda$ regular such that $f_{0}(X) \neq 0$. Moreover, there exists an integer $q \geq q(X)$ (see subsection 2.2 for the definition of $q(X)$ ) such that $f_{0}(X / q) \neq 0$. This follows from the fact that the polynomial in one variable $t$ given by $p(t):=f_{0}(t X)$ also cannot be identically zero, since $p(1)=f_{0}(X) \neq 0$. We set $\rho_{\ell}:=X / q$, and the lemma is proved.

Remark 4.4 Notice that the first vector $\xi_{1}$ in this basis is deliberately chosen so that the corresponding component $\mu^{\xi_{1}}$ is exactly the energy functional $E$. This is not necessary for the argument, but makes it evident how to apply a similar argument in Section 5 for just the energy functional.

Denote by $h_{1}=\left\langle\mu(\cdot), \xi_{1}\right\rangle, \ldots, h_{k}=\left\langle\mu(\cdot), \xi_{k}\right\rangle$ the components of the moment map $\mu$ corresponding to the $\xi_{k}$. By Proposition 2.9, each $h_{j}$ satisfies condition (C) of PalaisSmale with respect to the $H^{1}$ metric on $\Omega_{1}(G)$. Also let $a_{1}=\left\langle a, \xi_{1}\right\rangle, \ldots, a_{k}=\left\langle a, \xi_{k}\right\rangle$ be the coordinates of $a$ with respect to the basis $\left\{\xi_{k}\right\}$. Note that

$$
\mu^{-1}(a)=h_{1}^{-1}\left(a_{1}\right) \cap \ldots \cap h_{k}^{-1}\left(a_{k}\right) .
$$

Let $x_{0}$ be a point in $\mu^{-1}(a)$, and fix $j \in\{1,2, \ldots, k\}$. We denote by $g($, $)$ the Kähler metric and by $\langle,\rangle_{1}$ the $H^{1}$ Riemannian metric on $\Omega_{1}(G)$. We also denote by $\nabla h_{j}$ the gradient vector field of $h_{j}$ with respect to the Kähler metric. Since $x_{0}$ is a regular value of $h_{j}$, we have that $\nabla h_{j}\left(x_{0}\right) \neq 0$, so by a continuity argument there exists an open neighbourhood $U$ of $x_{0}$ in $\Omega_{1}(G)$ and a positive number $M$ such that the vector field on $U$ defined as

$$
Y_{j}:=-\frac{1}{g\left(\nabla h_{j}, \nabla h_{j}\right)} \nabla h_{j}
$$


satisfies $\left\|Y_{j}(x)\right\|_{1} \leq M$ for any $x \in U$. We now show that $Y_{j}$ can be extended to a vector field on all of $\Omega_{1}(G)$, with bounded $H^{1}$-length. Consider a coordinate system around $x_{0}$ which maps $x_{0}$ to 0 . We may assume without loss of generality that the field $Y_{j}$ has bounded $H^{1}$-length on the ball $B(0, r)$ in this coordinate system. We consider a smooth function $g$ on the coordinate system which is equal to 1 on $B(0, r / 3)$ and 0 outside the ball $B(0, r / 2)$. Define the vector field $Y_{j}^{\prime}:=g Y_{j}$ on this coordinate system. By extending further by 0 to all of $\Omega_{1}(G)$, we may take $Y_{j}^{\prime}$ to be defined on all of $\Omega_{1}(G)$. We will need the following result.

Lemma 4.5 There exists an open neighbourhood $U_{0}$ of $x_{0}$ and a number $\varepsilon>0$ with the property that for each $j=1,2, \ldots, k$, there exists a one-parameter group of automorphisms $\Phi_{t}^{j}, t \in \mathbb{R}$, of $\Omega_{1}(G)$, such that for any $t \in(-\varepsilon, \varepsilon)$ and for any $x \in U_{0}$, we have

$$
h_{j}\left(\Phi_{t}^{j}(x)\right)=h_{j}(x)-t .
$$

The group $\Phi_{t}^{j}$ leaves $\Omega_{\mathrm{alg}}(G)$ invariant.

Proof Because the metric $H^{1}$ is complete and the vector field $Y_{j}^{\prime}$ on $\Omega_{1}(G)$ defined above has bounded $H^{1}$-length, we deduce that $Y_{j}^{\prime}$ is completely integrable (see [24, Corollary 9.1.5]). Let $\Phi_{t}^{j}, t \in \mathbb{R}$, be the flow given by $Y_{j}^{\prime}$. By continuity, there exists $\varepsilon>0$ and $U_{0} \subset U$ such that for any $t \in(-\varepsilon, \varepsilon)$ and any $x \in U_{0}$, we have

$$
\Phi_{t}^{j}(x) \in U \text {. }
$$

Consider the function

$$
t \mapsto \gamma_{j}(t):=h_{j}\left(\Phi_{t}^{j}(x)\right) .
$$

For $t \in(-\varepsilon, \varepsilon)$ and $x \in U_{0}$ we have

$$
\frac{d \gamma_{j}}{d t}=-g\left(\nabla h_{j}\left(\Phi_{t}^{j}(x)\right), \frac{1}{g\left(\nabla h_{j}\left(\Phi_{t}^{j}(x)\right), \nabla h_{j}\left(\Phi_{t}^{j}(x)\right)\right)} \nabla h_{j}\left(\Phi_{t}^{j}(x)\right)\right)=-1 .
$$

Since $\gamma_{j}(0)=h_{j}(x)$, we deduce that $\gamma_{j}(t)=h_{j}(x)-t$.

We now prove the last statement of the lemma. We will argue the result only for $j \geq 2$, and simply note that a similar argument can be used for $j=1$. In order to simplify the notation, we will assume that $\rho_{j}$ is in the positive Weyl chamber, so that $C_{\lambda}\left(\rho_{j}\right)=C_{\lambda}$ (see Proposition 2.6). From the construction of $Y_{j}^{\prime}$ preceding the lemma, we can see that for any $x \in \Omega_{1}(G)$, the vector $\left(Y_{j}^{\prime}\right)_{x}$ is a multiple of $\nabla h_{j}(x)$. In particular, if $C_{\lambda}$ is a Bruhat cell, then for any $x \in C_{\lambda}$, the vector $\left(Y_{j}\right)_{x}$ is in $T_{x} C_{\lambda}$ (see Proposition 2.6). The restriction of $Y_{j}$ to $C_{\lambda}$ has bounded $H^{1}$ length, hence it generates a 1-parameter group of diffeomorphisms $\tilde{\Phi}_{t}^{j}, t \in \mathbb{R}$, of $C_{\lambda}$ (again by [24, Corollary 9.1.5]). For 
any $x \in C_{\lambda}$, the curves $\widetilde{\Phi}_{t}^{j}(x)$ and $\Phi_{t}^{j}(x)$ are integral curves of $Y_{j}^{\prime}$ in the Hilbert manifold $\Omega_{1}(G)$ with the same initial condition at $t=0$. By [17, Chapter IV, Section 2, Theorem 2], we deduce that $\Phi_{t}^{j}(x)=\widetilde{\Phi}_{t}^{j}(x)$ for all $t \in \mathbb{R}$. In other words, $\Phi_{t}^{j}$ leaves the Bruhat cell $C_{\lambda}$ invariant, for any $\lambda \in \check{T}$. Hence it leaves $\Omega_{\mathrm{alg}}(G)$ invariant, as desired.

Let us consider the map $\pi_{j}: U_{0} \cap h_{j}^{-1}\left(a_{j}-\varepsilon, a_{j}+\varepsilon\right) \rightarrow h_{j}^{-1}\left(a_{j}\right)$ given by

$$
\pi_{j}(x)=\Phi_{h_{j}(x)-a_{j}}^{j}(x) .
$$

We wish to compose these maps $\pi_{j}$ in order to take a sequence in $\Omega_{\text {alg }}(G)$ to a sequence contained in $\mu^{-1}(a) \cap \Omega_{\mathrm{alg}}(G)$. Complications arise, however, in that there is no guarantee that a projection $\pi_{j}$ leaves $h_{i}^{-1}\left(a_{i}\right)$ invariant for $i \neq j$. In order to get around this difficulty, we will need the following lemma.

Lemma 4.6 For any $1 \leq j \leq k$, the map

$$
A_{j}(x, t):=\pi_{1} \circ \ldots \circ \pi_{j-1}\left(\Phi_{t}^{j}(x)\right)
$$

maps an open neighbourhood of $\left(x_{0}, 0\right)$ in $h_{1}^{-1}\left(a_{1}\right) \cap \ldots \cap h_{j}^{-1}\left(a_{j}\right) \times(-\varepsilon, \varepsilon)$ diffeomorphically onto an open neighbourhood of $x_{0}$ in $h_{1}^{-1}\left(a_{1}\right) \cap \ldots \cap h_{j-1}^{-1}\left(a_{j-1}\right)$.

Proof First of all, we note that $A_{j}$ is a well-defined map from a certain open neighbourhood of $\left(x_{0}, 0\right)$ in $h_{1}^{-1}\left(a_{1}\right) \cap \ldots \cap h_{j}^{-1}\left(a_{j}\right) \times(-\varepsilon, \varepsilon)$ to $h_{1}^{-1}\left(a_{1}\right) \cap \ldots \cap h_{j-1}^{-1}\left(a_{j-1}\right)$. We will show that the latter map is a local diffeomorphism at $\left(x_{0}, 0\right)$. To this end, we consider the map

$$
B_{j}(x, t):=\pi_{2} \circ \ldots \circ \pi_{j-1}\left(\Phi_{t}^{j}(x)\right)
$$

from an open neighbourhood of $\left(x_{0}, 0\right)$ in $h_{2}^{-1}\left(a_{2}\right) \cap \ldots \cap h_{j}^{-1}\left(a_{j}\right) \times(-\varepsilon, \varepsilon)$ to $h_{2}^{-1}\left(a_{2}\right) \cap \ldots \cap h_{j-1}^{-1}\left(a_{j-1}\right)$. We have $A_{j}=\left.\pi_{1} \circ B_{j}\right|_{h_{1}^{-1}\left(a_{1}\right) \cap \ldots \cap h_{j}^{-1}\left(a_{j}\right)}$ and consequently the following diagram is commutative:

$$
\begin{aligned}
& \left(\cap_{\ell=2}^{j} \operatorname{ker}\left(d h_{\ell}\right)_{x_{0}}\right) \oplus \mathbb{R} \stackrel{\left(d B_{j}\right)_{\left(x_{0}, 0\right)}}{\longrightarrow} \cap_{\ell=2}^{j-1} \operatorname{ker}\left(d h_{\ell}\right)_{x_{0}} \\
& \imath \uparrow \quad \downarrow\left(d \pi_{1}\right)_{x_{0}} \\
& \left(\cap_{\ell=1}^{j} \operatorname{ker}\left(d h_{\ell}\right)_{x_{0}}\right) \oplus \mathbb{R} \stackrel{\left(d A_{j}\right)_{\left(x_{0}, 0\right)}}{\longrightarrow} \cap_{\ell=1}^{j-1} \operatorname{ker}\left(d h_{\ell}\right)_{x_{0}}
\end{aligned}
$$

Since $\left(d B_{j}\right)_{\left(x_{0}, 0\right)}(v, 0)=v$, we only need to prove that $\left(d A_{j}\right)_{\left(x_{0}, 0\right)}(0,1) \notin \operatorname{ker}\left(d h_{j}\right)_{x_{0}}$. This is true, because Lemma 4.3 (b) says that

$$
g\left(\nabla\left(h_{j}\right)_{x_{0}},\left(d \pi_{1}\right)_{x_{0}} \circ \ldots \circ\left(d \pi_{j-1}\right)_{x_{0}}\left(\nabla\left(h_{j}\right)_{x_{0}}\right)\right) \neq 0 .
$$


We may now prove the main result of this section.

Proof of Proposition 4.1 Let $x_{0} \in \mu^{-1}(a)$. We wish to show that $\mu^{-1}(a) \cap \Omega_{\mathrm{alg}}(G)$ is dense in $\mu^{-1}(a)$. To this end it would suffice to construct a sequence $y(r) \in$ $\mu^{-1}(a) \cap \Omega_{\text {alg }}(G)$ such that $y(r) \rightarrow x_{0}$ in the $H^{1}$ topology. By [26, Proposition 3.5.3], we know that $\Omega_{\mathrm{alg}}(G)$ is dense in $\Omega_{1}(G)$, so there exists a sequence $x_{0}(r)$ with $x_{0}(r) \in \Omega_{\text {alg }}(G), x_{0}(r) \rightarrow x_{0}$. Then

$$
x_{1}(r):=\pi_{1}\left(x_{0}(r)\right)
$$

has the property $x_{1}(r) \in h_{1}^{-1}\left(a_{1}\right) \cap \Omega_{\mathrm{alg}}(G)$ and $x_{1}(r) \rightarrow \pi_{1}\left(x_{0}\right)=x_{0}$. Note that since $x_{0} \in h_{i}^{-1}\left(a_{i}\right) \quad \forall i$, the projection $\pi_{i}$ fixes $x_{0}$ for all $i$. Suppose now we have a sequence $x_{\ell}(r) \rightarrow x_{0}$, where $x_{\ell}(r) \in h_{1}^{-1}\left(a_{1}\right) \cap \cdots \cap h_{\ell}^{-1}\left(a_{\ell}\right)$. From Lemma 4.6, $A_{\ell}$ is a local diffeomorphism, and hence by taking an appropriate subsequence, we may define $x_{\ell+1}(r)$ by the relation

$$
x_{\ell}(r)=A_{\ell}\left(x_{\ell+1}(r), t_{r}\right)
$$

where $x_{\ell+1}(r) \in h_{1}^{-1}\left(a_{1}\right) \cap \cdots \cap h_{\ell+1}^{-1}\left(a_{\ell+1}\right) \cap \Omega_{\mathrm{alg}}(G)$. Because $A_{\ell}$ is a local diffeomorphism around $\left(x_{0}, 0\right)$ (see Lemma 4.6), from $A_{\ell}\left(x_{\ell+1}(r), t_{r}\right) \rightarrow A_{\ell}\left(x_{0}, 0\right)$ we deduce $x_{\ell+1}(r) \rightarrow x_{0}$. Continuing, we may set $y(r):=x_{k}(r)$ (recall that $k-1$ is the dimension of $T$ ). In this way Proposition 4.1 is completely proved.

We may immediately conclude that Theorem 1.2 holds.

Proof of Theorem 1.2 We have just shown that $\mu^{-1}(a) \cap \Omega_{\mathrm{alg}}(G)$ is dense in $\mu^{-1}(a) \subseteq \Omega_{1}(G)$ in the $H^{1}$ topology. Since $\mu^{-1}(a) \cap \Omega_{\mathrm{alg}}(G)$ is connected in the direct limit topology on $\Omega_{\mathrm{alg}}(G)$, and the direct limit topology on $\Omega_{\mathrm{alg}}(G)$ is finer than the subset topology induced from the $H^{1}$ topology on $\Omega_{1}(G)$ (see Proposition 2.1), we may conclude that $\mu^{-1}(a) \cap \Omega_{\mathrm{alg}}(G)$ is also connected in the $H^{1}$ topology, considered as a subset of $\Omega_{1}(G)$. The closure of the connected set is also connected, and $\mu^{-1}(a)$ is closed, so we conclude that $\mu^{-1}(a)$ is connected, as desired.

By repeating the same argument using only the function $h_{1}=E$, we also immediately obtain the following, which is a special case of Theorem 1.3.

Proposition 4.7 For any $a \in \mathbb{R}$ which is a regular value of $E: \Omega_{1}(G) \rightarrow \mathbb{R}$, the preimage $E^{-1}(a)$ is empty or connected. 


\section{The singular levels of the energy on $\Omega_{1}(G)$}

Proposition 4.7 of the previous section already proves the connectivity for level sets of regular values for the energy functional. Hence, in order to prove completely Theorem 1.3 , it only remains to consider the case of a singular level set of the energy functional $E$. We prove this using essentially the same ideas as in Section 4, except that we must remove a certain subset of the critical points in order to force the regularity condition. It is then also necessary to prove that the condition (C) of Palais-Smale holds even after we have removed this subset. This is the main technical result of this section.

We first observe that, as in Section 4, it suffices to prove the following.

Proposition 5.1 Let $a \in \mathbb{R}$ be a singular value of $E: \Omega_{1}(G) \rightarrow \mathbb{R}$. Then $E^{-1}(a) \cap$ $\Omega_{\mathrm{alg}}(G)$ is dense in $E^{-1}(a)$.

Following the ideas of the previous section, we will prove below that if $x_{0} \in E^{-1}(a)$, then there exists a sequence $\{x(r)\}$ with $x(r) \in E^{-1}(a) \cap \Omega_{\text {alg }}(G)$ and such that $x(r) \rightarrow x_{0}$. Denote by $K$ the (closed) subset of $\mu^{-1}(a)$ consisting of all singular points of $E$ contained in $E^{-1}(a)$. This $K$ is of the type $\Lambda_{|\lambda|}$ in Proposition 2.7, so it is contained in $\Omega_{\mathrm{alg}}(G)$. We may assume without loss of generality that $x_{0} \notin K \subseteq$ $\Omega_{\mathrm{alg}}(G)$, since otherwise there trivially exists a sequence in $\Omega_{\mathrm{alg}}(G)$ converging to $x_{0}$ (eg, the constant sequence).

We now fix, for the rest of the discussion, such a point $x_{0} \in E^{-1}(a), x_{0} \notin K$. We must show that singular values of the energy functional are well-behaved. We have the following two lemmas.

Lemma 5.2 The critical values of $E: \Omega_{1}(G) \rightarrow \mathbb{R}$ are isolated in $\mathbb{R}$.

Proof As shown in [2], the critical values are

$$
E(\alpha)=\frac{1}{2}|\alpha|^{2},
$$

where $\alpha$ is in the integer lattice $\check{T}=\operatorname{ker}(\exp : \mathfrak{t} \rightarrow T) \subset \mathfrak{t}$. We claim that the set all $E(\alpha)$ from above has no limit points. Suppose not; then such a limit point would occur within a ball of radius $R<\infty$. But $\{X \in \mathfrak{t}:|X| \leq R\}$ is compact, and therefore only contains finitely many points in the integer lattice $\check{T}$.

Lemma 5.3 There exists a closed neighborhood $K_{0}$ of $K$ in $\Omega_{1}(G)$ such that $x_{0} \notin K_{0}$ and the only critical points of $E$ in $K_{0}$ are those in $K$. 
Proof There exist open neighborhoods $U$ (respectively $V$ ) of $K$ (respectively of $x_{0}$ ) in $\Omega_{1}(G)$ such that $U \cap V=\phi$ (because $\Omega_{1}(G)$ is a normal topological space). We choose $\varepsilon$ such that the only critical points in $E^{-1}([a-\varepsilon, a+\varepsilon])$ are those in $E^{-1}(a)$ (see Lemma 5.2). We define $K_{0}$ the closure of $U \cap E^{-1}(a-\varepsilon, a+\varepsilon)$.

We consider the infinite dimensional manifold $M:=\Omega_{1}(G) \backslash K_{0}$ (which is a Hilbert manifold) and the restriction of $E$ to it.

Lemma 5.4 The function $E$ restricted to $M$ satisfies condition $(C)$ with respect to the restriction of the $H^{1}$ metric to $M$.

Proof Condition (C) is given in Definition 2.8. Let $\{s(n)\}$ be a sequence in $M$ with $|E(s(n))|$ bounded and $\left\|\nabla E_{s(n)}\right\|_{1} \rightarrow 0$. Since $E: \Omega_{1}(G) \rightarrow \mathbb{R}$ does satisfy $(\mathrm{C})$, we deduce that there exists $p \in \Omega_{1}(G)$ with $s\left(n_{k}\right) \rightarrow p$. But $p$ cannot be in $K_{0}$. Indeed, $p$ is necessarily a critical point, so if $p \in K_{0}$, then $p \in K$ (as in the statement of Lemma 5.3); this is not possible, because all $s\left(n_{k}\right)$ are contained in the complement of a neighborhood of $K$, hence the limit must stay out of that neighborhood. The lemma is proved.

We may now prove the proposition.

Proof of Proposition 5.1 For the function $E$ restricted to $M, a$ is a regular value. By using the methods from Section 4 , we can construct a sequence $\{x(r)\}$ with $x(r) \in \Omega_{\mathrm{alg}}(G) \cap M \cap E^{-1}(a)$ and $x(r) \rightarrow x_{0}$. The crucial fact is that $\nabla E$ is always tangent to Bruhat cells, hence the flow $\Phi_{t}$ of $E$ leaves $M \cap \Omega_{\mathrm{alg}}$ invariant.

We assemble these observations to finish the proof of Theorem 1.3.

Proof of Theorem 1.3 Since Proposition 4.7 already proves Theorem 1.3 for the case of regular values, we only need to discuss the case when $a$ is a singular value. In this case, we use Proposition 3.5 and Proposition 5.1 and an argument exactly analogous to that given for Theorem 1.2 at the end of Section 4.

\section{References}

[1] MF Atiyah, Convexity and commuting Hamiltonians, Bull. London Math. Soc. 14 (1982) 1-15 MR642416

[2] M F Atiyah, A N Pressley, Convexity and loop groups, from: "Arithmetic and geometry, Vol. II”, Progr. Math. 36, Birkhäuser, Boston (1983) 33-63 MR717605 
[3] T Bröcker, T tom Dieck, Representations of compact Lie groups, Graduate Texts in Mathematics 98, Springer, New York (1985) MR781344

[4] I Dolgachev, Lectures on invariant theory, London Mathematical Society Lecture Note Series 296, Cambridge University Press, Cambridge (2003) MR2004511

[5] D S Freed, The geometry of loop groups, J. Differential Geom. 28 (1988) 223-276 MR961515

[6] M A Guest, A N Pressley, Holomorphic curves in loop groups, Comm. Math. Phys. 118 (1988) 511-527 MR958810

[7] V Guillemin, S Sternberg, Convexity properties of the moment mapping, Invent. Math. 67 (1982) 491-513 MR664117

[8] V Guillemin, S Sternberg, Multiplicity-free spaces, J. Differential Geom. 19 (1984) 31-56 MR739781

[9] M Harada, A Henriques, T S Holm, Computation of generalized equivariant cohomologies of Kac-Moody flag varieties, Adv. Math. 197 (2005) 198-221 MR2166181

[10] P Heinzner, L Migliorini, Projectivity of moment map quotients, Osaka J. Math. 38 (2001) 167-184 MR1824905

[11] L C Jeffrey, F C Kirwan, Localization for nonabelian group actions, Topology 34 (1995) 291-327 MR1318878

[12] Y Karshon, E Lerman, The centralizer of invariant functions and division properties of the moment map, Illinois J. Math. 41 (1997) 462-487 MR1458185

[13] F C Kirwan, Cohomology of quotients in symplectic and algebraic geometry, Mathematical Notes 31, Princeton University Press, Princeton, NJ (1984) MR766741

[14] F Kirwan, Rational intersection cohomology of quotient varieties II, Invent. Math. 90 (1987) 153-167 MR906583

[15] R R Kocherlakota, Integral homology of real flag manifolds and loop spaces of symmetric spaces, Adv. Math. 110 (1995) 1-46 MR1310389

[16] S Kumar, Kac-Moody groups, their flag varieties and representation theory, Progress in Mathematics 204, Birkhäuser, Boston (2002) MR1923198

[17] S Lang, Differential manifolds, Addison-Wesley Publishing Co.,, Reading, MALondon-Don Mills, Ont. (1972) MR0431240

[18] E Lerman, Symplectic cuts, Math. Res. Lett. 2 (1995) 247-258 MR1338784

[19] W Liu, Convexity of moment polytopes of algebraic varieties, Proc. Amer. Math. Soc. 131 (2003) 2921-2932 MR1974350

[20] A-L Mare, Connectivity and Kirwan surjectivity for isoparametric submanifolds, Int. Math. Res. Not. (2005) 3427-3443 MR2204640

[21] S A Mitchell, A filtration of the loops on SU(n) by Schubert varieties, Math. Z. 193 (1986) 347-362 MR862881 
[22] D Mumford, J Fogarty, F Kirwan, Geometric invariant theory, third edition, Ergebnisse series 34, Springer, Berlin (1994) MR1304906

[23] RS Palais, Morse theory on Hilbert manifolds, Topology 2 (1963) 299-340 MR0158410

[24] R S Palais, C-L Terng, Critical point theory and submanifold geometry, Lecture Notes in Mathematics 1353, Springer, Berlin (1988) MR972503

[25] A N Pressley, The energy flow on the loop space of a compact Lie group, J. London Math. Soc. (2) 26 (1982) 557-566 MR684568

[26] A N Pressley, G B Segal, Loop groups, Oxford Mathematical Monographs, The Clarendon Press Oxford University Press, New York (1986) MR900587, Oxford Science Publications

[27] C-L Terng, Proper Fredholm submanifolds of Hilbert space, J. Differential Geom. 29 (1989) 9-47 MR978074

[28] C-L Terng, Convexity theorem for infinite-dimensional isoparametric submanifolds, Invent. Math. 112 (1993) 9-22 MR1207475

[29] S Tolman, J Weitsman, The cohomology rings of symplectic quotients, Comm. Anal. Geom. 11 (2003) 751-773 MR2015175

[30] C T Woodward, The Yang-Mills heat flow on the moduli space of framed bundles on a surface, Amer. J. Math. 128 (2006) 311-359 MR2214895

Department of Mathematics and Statistics, McMaster University, 1280 Main Street West Hamilton, Ontario L8S 4K1, Canada

Department of Mathematics, 589 Malott Hall, Cornell University

Ithaca, NY 14850-4201, USA

Department of Mathematics, University of Toronto, Toronto, Ontario M5S 2E4, Canada Department of Mathematics and Statistics, University of Regina, College West 307.14 Regina, Saskatchewan S4S 0A2, Canada

Megumi.Harada@math.mcmaster.ca, tsh@math.cornell.edu, jeffrey@math.toronto.edu, mareal@math.uregina.ca

Proposed: Ralph Cohen

Received: 4 April 2005

Seconded: Haynes Miller, Frances Kirwan

Revised: 15 July 2005 